\title{
An Intelligent Marshalling Model for Enterprise Station Freight Railway
}

\author{
Junxia GUO ${ }^{\mathrm{a}}$, Gang $\mathrm{LU}^{\mathrm{a}, 1}$, Zili Xie ${ }^{\mathrm{b}}$, Jiawei $\mathrm{WEN}^{\mathrm{c}}$, Nanshan XU ${ }^{\mathrm{a}}$ \\ ${ }^{\mathrm{a}}$ College of Information Science and Technology, Beijing University of Chemical \\ Technology, Beijing 100029, China \\ ${ }^{b}$ Research and Development Center for Science \& Technology, SINOPEC Pipeline \\ Storage \& Transportation Company limited, Xuzhou 221008, China \\ ${ }^{\mathrm{c}}$ China Science and Technology Museum, Beijing 100029, China
}

\begin{abstract}
Railway marshalling and transportation is an important component of the production supply chain for large and medium-sized enterprises in China. Traditional inefficient manual-made marshalling plans usually are not optimal in time or energy consuming. An efficient method needs to be developed to find the optimal marshalling plans automatically. This paper mainly studies the railway train automatic marshalling in large and medium-sized enterprises in China. Based on the investigation at the train station of a certain enterprise, according to the railway track information, carriage information, and production task information, this paper designs the abstracted railway state definitions of the station. Then based on the state definitions, the scheduling rules, and the objective function of time cost and economic cost, this paper converts abstract scheduling instructions into a general railway automatic marshalling model which can be executed by computers. By introducing the greedy strategies into different situations to optimize the algorithm of tracks occupation, carriages selection and train path selection in the model, the planning efficiency can be improved while ensuring the economic benefits of the enterprises and the quality of the formation plan. The experimental results show that the proposed model can generate fewer marshalling plans and find the optimal one faster in most cases, which proves the feasibility and availability of the model.
\end{abstract}

Keywords. enterprise station railway, scheduling methods and constraint sets, automatic marshalling model

\section{Introduction}

As the source of freight railway transportation, railway marshalling is the first step to ensure the normal operation of railway stations. The railway stations in China mainly serve large and medium-sized industrial areas such as mining, petroleum, petrochemical plants, and metallurgical processing industries. With the development of the national economy, the railway freight volume of stations is increasing, the marshalling time is more compact, and the dispatching operation is more complicated. A marshalling operation scheduling includes freight train arrival, dispatch, and marshalling operations. There are always some constrains for a good train marshalling plan, such as the number of hooking or unhooking operations or moving distance of the carriages should be as less

\footnotetext{
${ }^{1}$ Corresponding Author: Gang LU, College of Information Science and Technology, Beijing University of Chemical Technology, Beijing 100029, China; E-mail: lugang@mail.buct.edu.cn

The work described in this paper is supported by the National Natural Science Foundation of China under Grant No.61702029.
} 
as possible, or there are some rules of using the tracks and carriages because of their different functions. For example, carriages loading chemicals A must not be connected with carriages loading chemicals B because it's dangerous. Also, maybe some tracks can be only used for carriages storage but not moving them, or vice versa. All those constrains make us have to consider the availability of the marshalling plans as well as their time and energy consuming. However, making marshalling plans under complex constrains manually usually is slow and inefficient. Optimized plans cannot be guaranteed by manually-made plans in respect of the time and energy consuming of the plans.

Train scheduling and marshalling problem is a typical NP problem [1]. In the field of mathematical modeling, Dessouky [2] regarded the railway freight route as a network with different scales, the route planning problem as the core of the train scheduling research, the minimum delay as the evaluation indicator, and adopted different heuristic algorithms to optimize the scheduling route in the large and medium-sized networks. Krasemann [3] and He [4] respectively apply the greedy algorithm to reduce data interference and avoid train interference, so as to implement rapid rescheduling of trains. Pathania et. al [5] proposed a greedy strategy for multi-core scheduling, which is based on dynamic programming scheduling problems to reduce scheduling overhead in space searching and data filtering. Katsavounis [6] used the graph theory to model the production scheduling problems, and greedy strategy to solve the allocation problems of limited resources in the network with topology structure. Ribas et. al [7] applied the improved iterative greedy algorithm to the scheduling optimization of pipeline jobs. By introducing a genetic algorithm, He et. al [8] solved the fuzzy scheduling problem in the railway management system. Chuntian Zhang et. al [9] propose a joint optimization model for a railway network with double-track, where the upstream and downstream trains are independent and a maintenance task on a section cannot be split or disrupted. A heuristic algorithm using Lagrangian relaxation is developed in their paper. Due to the large number of constraints, they used a dynamic constraint-generation technique in the iterations of the sub-gradient optimization procedure. In [10], Chuntian Zhang et. al researched integrated optimization of train scheduling and maintenance planning on high-speed railway corridors. They used some linearization techniques to formulate a mixed integer linear programming model to identify the operation modes and the timetable of SDSA-trains, by integrating the time window selection of regular maintenances on high-speed railways. They introduced state variables to indicate whether a train is running on high-speed railway or not. That made it convenient to express the selection of operation modes.

In the field of computer intelligent marshalling, most of the research takes national railway stations [11] or metro lines [12] as the research object. The research and application on medium and large-scale enterprise stations are weak. The existing intelligent dispatching system cannot be directly applied to station railways for those enterprise stations because that most of those researches focus on the multi-trains dispatching between stations instead of multi-track scheduling in one station for multifunctional carriages. Therefore, it is necessary to research and develop automatic marshalling models suitable for large and medium-sized enterprise station railways in one station with complex constrains of tracks and carriages.

The common goal of an automatic marshalling model is to organize a new train as soon as possible and prepare for departure at the minimum cost on the premise of meeting the planning requirements. Because the train scheduling process involves a large number of variables and multiple rules, which should be transformed into constraints of 
the model, the train marshalling scheduling problem is essentially a large-scale combinatorial optimization problem. This paper mainly studies the following problems:

(1) Designing the state definition of stations. By analyzing the specific content and process of operational railway dispatching, design the state information of the station.

(2) Designing the automatic train marshalling model. According to the real-time states of the station, the model can automatically compile the scheduling scheme by scheduling constraints, which meet the planning requirements.

(3) Aiming at the problem of marshalling time cost and economic benefit of enterprises, different greedy strategies are introduced to optimize the general marshalling model so that the marshalling time of the obtained scheduling scheme is the shortest and the launching revenue is the largest.

\section{Station State Definitions}

Before solving the railway marshalling problem, it is necessary to define the variables that affect the marshalling planning. The station state information is defined from three aspects, which are railway carriages, tracks, and marshalling tasks. The state space is represented by $U=<C, P, A>$, where $C$ is the carriage state, $P$ is the track state, and $A$ is the marshalling task state. $U$ can represent all the carriages and tracks in the station railway at a certain time, as well as the marshalling task information of the station.

\section{1) Carriages State}

The state of railway carriages in the station is composed of four types of information: basic information, position of the carriage, availability of the carriage, and the working state, as shown in Table $1 . C_{\mathrm{v}}$ refers to the availability state of the carriage. $C_{\mathrm{v}} \in\{1,0\}$ indicates whether there is any follow-up plan for the carriage. $C_{\mathrm{o}}$ represents the carriage's working state. $C_{\mathrm{o}} \in\{1,2,3\}$ respectively represent the three working states of railway carriages in the station during the stage planning cycle. When $C_{\mathrm{o}}=1$, the carriage is in the free state, which means that the carriage has completed all the previous tasks and is placed on the respective track, which can be directly invoked. When $C_{\mathrm{o}}=2$, the carriage is in the waiting loading and unloading state. For example, the carriage has arrived at the station, and is waiting for the unloading operation after the disassembly. When $C_{\mathrm{o}}=3$, the carriage is in running state, which means the carriage is about to arrive at the station as planned and has been registered on the dispatch schedule.

Table 1. Symbol description of carriage state

\begin{tabular}{ccl}
\hline Type & Symbol & \multicolumn{1}{c}{ Definition } \\
\hline Basic information & $C$ & Carriage set \\
& $C_{\mathrm{n}}$ & Wagon number of carriage \\
& $C_{\mathrm{k}}$ & Kind of carriage kind \\
& $C_{\mathrm{c}}$ & Commodity of carriage \\
& $C_{\mathrm{a}}$ & Carriage attribution \\
& $C_{\mathrm{w}}$ & Carriage load \\
Position state & $C_{\mathrm{m}}$ & Carriage charges \\
& $C_{\mathrm{t}}$ & Parking track of carriage \\
Availability state & $C_{\mathrm{l}}$ & Carriage positioning on track \\
Working state & $C_{\mathrm{v}}$ & Availability of carriage \\
\hline
\end{tabular}

\section{2) Track State}

There are $m$ tracks in the station, denoted by $P=\left\{p_{1}, p_{2} \ldots, p_{\mathrm{m}}\right\}$. If $p_{\mathrm{i}}$ has $j$ connected track, then all states of track $p_{\mathrm{i}}$ are expressed as: $p_{\mathrm{i}}=<P_{\mathrm{t}}, P C_{\mathrm{w}}, P F_{\mathrm{i}}, L>$, where $L=\left\{<p_{1}\right.$, $\left.L_{\mathrm{t} 1}>,<p_{2}, L_{\mathrm{t} 2}>\ldots,<p_{\mathrm{j}}, L_{\mathrm{tj}}>\right\} . P F_{\mathrm{i}} \in\{1,2,3,4,5\}$ is the track function set of the station, representing the five types of functions in the station's track. $P F_{1}$ means the track is an 
arrival-marshalling line, which means trains can be marshalled, disassembled, dispatched and temporarily parked on it. $P F_{2}$ means the track is a preparatory emergency task line, and this track only performs the marshalling and dispatching of emergency priority trains. $P_{3}$ means the track is a parking traction line, which is used for parking existing carriages that have been loaded and unloaded or temporarily parking carriages. $P F_{4}$ means the track is a loading line, while $P F_{5}$ means the track is an unloading line. Those two kinds of tracks are respectively used for different specific commodity loading and unloading. Table 2 lists the symbol descriptions of track state.

Table 2. Symbol descriptions of track state

\begin{tabular}{ccl}
\hline Type & Symbol & Definition \\
\hline Track basic information & $P$ & Track set \\
& $P_{\mathrm{t}}$ & Track name \\
& $P C_{\mathrm{w}}$ & Track carriage capacity \\
Adjacent track information & $P F_{\mathrm{i}}$ & Track function \\
& $L$ & Adjacent tracks set \\
& $L_{\mathrm{t}}$ & Trains run time between adjacent tracks \\
\hline
\end{tabular}

\section{3) Marshalling Task}

The preparation of the marshalling plan requires information of the production tasks in a certain period, such as the number of marshalling and the type of carriages. The involved symbols and their description are shown in Table 3.

Table 3. Symbol description of marshalling task

\begin{tabular}{cl}
\hline Symbol & Definition \\
\hline$A$ & Production task set \\
$A_{\mathrm{n}}$ & Task number \\
$A_{\mathrm{d}}$ & Task destination \\
$A_{\mathrm{k}}$ & Task type \\
$A_{\mathrm{c}}$ & Task commodity \\
$A_{\mathrm{a}}$ & Task Quantity \\
$A_{\mathrm{t}}$ & Task requires carriages number \\
$A_{\mathrm{p}}$ & Task quotation \\
$T_{\mathrm{D}}$ & Production task cycle \\
\hline
\end{tabular}

In this paper, three types of tasks are defined, which are denoted as $A_{\mathrm{k}} \in\{1,2,3\}$. They respectively represent product delivery task, empty vehicle returning task, and urgent priority task. The arrival and departure marshalling tracks can be used for product delivery and empty vehicle returning tasks. Emergency priority tasks are grouped separately on the preparatory emergency task tracks. They are not subject to the lower limit of starting load, which means as soon as the marshalling completes, the train can apply for starting.

\section{Construction of Marshalling Model}

The automatic marshalling model mainly considers three issues: the selection of marshalling carriages, the occupation of marshalling tracks and temporary dispatching tracks, and the operation of carriages.

Basing on the definitions in Section 2, two assumptions are made as follows:

Assumption 1: the time spent on hooking and unhooking is ignored;

Assumption 2: carriages move with uniform speed.

\subsection{Selection of Marshalling Carriages}

\section{1) Rule of Selecting Carriage}


When selecting carriages, three issues should be considered: the corresponding commodity, availability state of the carriages, and the number of carriages required. The carriages selection algorithm is shown in Algorithm 1.

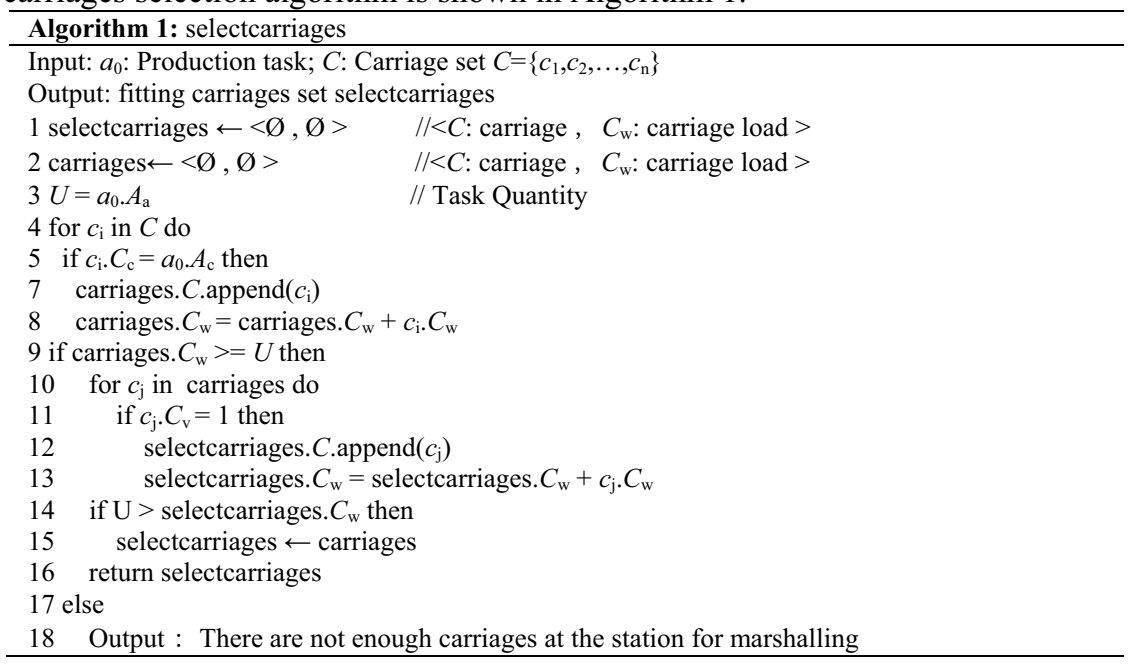

\section{2) Rules for Grouping Carriages}

The basic operations of railway dispatching are hooking and unhooking. Too many hooking or unhooking operations not only reduce the efficiency of station dispatching operation, but also lead to increased wear and tear of equipment and workload. A good marshalling scheme should take hooking and unhooking operations as less as possible to form a carriage group by combining carriages with the same state.

By this rule, after selecting marshalling carriages, all the suitable carriages parking on the same track will be interconnected into one carriage group. All the carriages groups in the station will be recorded, including their numbers and total load. The symbol descriptions of carriage groups are listed in Table 4.

Table 4. Symbol Description of Carriage Group

\begin{tabular}{cl}
\hline Symbol & Description \\
\hline$G$ & Carriage group set \\
$G_{\mathrm{n}}$ & Carriage group number \\
$G_{\mathrm{c}}$ & Carriage group category \\
$G_{\mathrm{w}}$ & Carriage group load \\
$G_{1}$ & Carriage amount of carriage group \\
$G_{\mathrm{t}}$ & Parking track of carriage group \\
\hline
\end{tabular}

If there are $n$ groups of carriages in the station, the set of them is denoted by $G=\left\{g_{1}, g_{2}, \ldots, g_{\mathrm{n}}\right\}$. A certain carriage group $g_{\mathrm{i}}$ contains $m$ carriages, where $g_{\mathrm{i}}=\left\{c_{1}, c_{2}, \ldots, c_{\mathrm{m}}\right\}$. In addition, carriage group information is expressed as $g_{\mathrm{i}}=\left\{G_{\mathrm{n}}, G_{\mathrm{c}}, G_{\mathrm{w}}, G_{\mathrm{l}}, G_{\mathrm{t}}\right\}$.

\subsection{Parking Rule of Disassembling Train}

Before disassembling or marshalling a train, it is necessary to decide the operating track. In order to ensure the transportation safety of station, different traction lines are usually preset for different functions, such as train disassembling, product delivery, or empty carriage storage, and so on. At the same time, during disassembling, traffic distribution, or other operations, it is often necessary to temporarily move away the carriages those do not participate in the subsequent operations. Only the arrival and departure marshalling 
traction lines and the carriage storage traction lines can be temporarily assigned for parking the trains.

\subsection{General Marshalling Model}

The general marshalling model is proposed by summarizing the actual marshalling planning process and constrains. Basing on state space searching, exhaustive method is applied here to find the optimal solution. In order to avoid invalid state searching, constrains are introduced into the searching progress to prune the invalid states. The general marshalling model is as follows.

Step 1: Initialize station status, including station track, carriage status information, and production task.

Step 2: Select the marshalling carriage. According to the requirements of production task, and the method of scheduling and carriage selection in the constraint set, enumerate all the suitable carriages and carriage groups, and then decide the information such as load and position.

Step 3: Generate the following marshalling plan:

(1) Deciding the tracks to use: decide the operation tracks for different production tasks, the temporary scheduling tracks may be involved, and the target group tracks.

(2) Generating train scheduling plan: simulate the scheduling plan by moving the selected carriage groups to the target tracks, and calculate the time cost and economic cost. Meanwhile, the train position and working status are updated.

Step 4: Select optimal marshalling scheme. By comparing the generated marshalling schemes by Step 3 in their time cost and economic cost, the optimal marshalling scheme can be selected.

Step 5: Successively generate marshalling plans. If there are more than one production and transportation tasks, take the current state of the station after previous optimal marshalling plan as the initial state of the next plan, and then execute Step 1 to Step 4 again.

\subsection{Optimized Marshalling Model by Greedy Strategies}

General marshalling model proposed in Section 3.3 can find the optimal solution by exhaustive searching under constrains. However, when the number or types of carriages becomes larger, or the topology of the tracks becomes complex, the state space will expand rapidly. That will make general marshalling model inefficient. There are some NP problems involved in the general marshalling model, such as the backpack problem in selecting the trains and carriages for capacity, the routing problem in selecting the trains' running route and the temporarily parking track, etc.. As a result, greedy strategy is introduced into those parts to try to improve the model. Although greedy strategy may not provide the exact optimal solution, it still helps to approach to the optimal solution, as well as to improve the efficiency.

Concretely, greedy strategy is introduced in three parts: 1) choosing the best carriage groups based on the biggest capacity and the best position; 2) getting the shortest working time $T_{\mathrm{c}} ; 3$ ) optimizing the running path based on trains' running time minimization. Based on those greedy strategies, the optimized marshalling model is designed to prepare the optimal marshalling plan in a shorter time by generating less marshalling plans. The process steps are similar with the general marshalling model, except the using of above greedy strategies. 


\section{Experiment and Performance Analysis}

This paper uses the railway station of a certain supply and marketing department of a company in China as the experimental example. The railway line of this station is Class I Railroad. Its allowable speed is $30 \mathrm{~km} / \mathrm{h}$. The layout of the tracks is shown in Figure 1. The text in the figure indicates the code name of the station track. This station is an internal marshalling station of the enterprise, which mainly handles the dissembling of freight trains and the dispatch of product trains. There are 28 tracks, 84 carriages, 7 marshalling tasks, and 1 available train in the experimental environment.

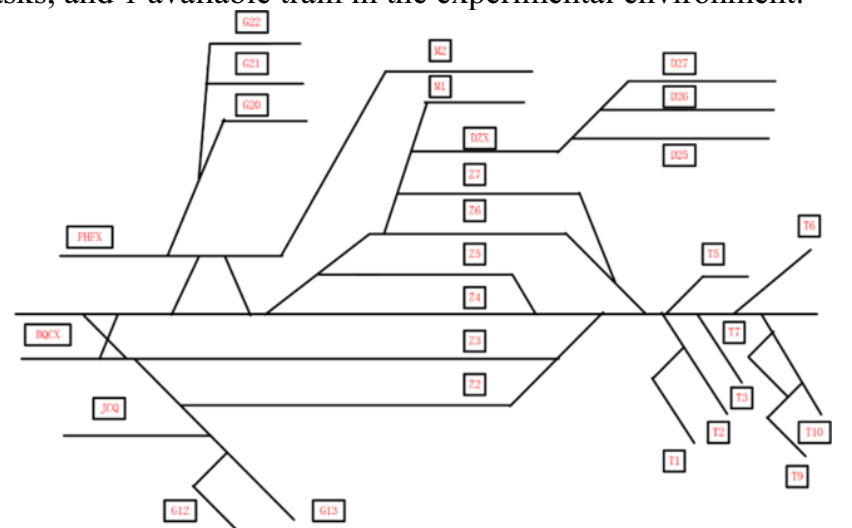

Figure 1. Layout of the tracks in the railway station of supply and Marketing Department

The marshalling tasks received by the station on a certain working day are taken as the test data. The state of the station at that time is taken as the initial environments. The general marshalling model and the optimized marshalling model are compared on the experimental environments. The experimental results are shown in Table 5.

Table 5. Comparative Experimental Results

\begin{tabular}{ccccc}
\hline & \multicolumn{2}{c}{ General marshalling model } & \multicolumn{2}{c}{$\begin{array}{c}\text { Optimized marshalling model by greedy } \\
\text { strategies }\end{array}$} \\
\cline { 2 - 5 } Tasks & $\begin{array}{c}\text { \# of marshalling plans } \\
\text { generated in one task }\end{array}$ & $\begin{array}{c}\text { Optimal } \\
\text { marshalling time } \\
(\mathrm{min})\end{array}$ & $\begin{array}{c}\text { \# of marshalling plans } \\
\text { generated in one task }\end{array}$ & $\begin{array}{c}\text { Optimal } \\
\text { marshalling time } \\
\text { (min) }\end{array}$ \\
\hline Task 1 & 4 & 35 & $\mathbf{1}$ & $\mathbf{3 5}$ \\
Task 2 & 2 & 75 & $\mathbf{1}$ & $\mathbf{7 5}$ \\
Task 3 & 7 & 35 & $\mathbf{1}$ & $\mathbf{3 5}$ \\
Task 4 & 2 & 40 & $\mathbf{2}$ & $\mathbf{4 0}$ \\
Task 5 & 13 & $\mathbf{3 0}$ & $\mathbf{2}$ & 45 \\
Task 6 & 762 & 70 & $\mathbf{3}$ & $\mathbf{7 0}$ \\
Task 7 & 63 & 45 & $\mathbf{4 5}$ \\
\hline
\end{tabular}

In Table 5, it can be found that both the two automatic marshalling models designed in this paper can effectively obtain the optimal marshalling plan of stations, which proves the correctness of state descriptions designed in this paper. According to the experimental comparison between the general marshalling model and the optimized model, the optimized model needs to generate less plans than the general model in order to find the optimal marshalling plan with shortest time. Along with the increasing number of carriages in different working states in the station, the general marshalling model using exhaustive method needs to explore significantly increasing number of plans. In that case, the advantage of the optimized model is more distinct in generating 
much less plans. For example, the general model need to explore all the 2 plans for Task 2 , while the optimized model generates 1 plans with the same marshalling time of 75 minutes. In contrast with Task 2, the general model needs to generate all the 762 plans for Task 6, and the optimal plan takes 70 minutes for marshalling. However, by the optimized model, only 15 plans are generated to find the optimal one with the same 70 minutes of marshalling time. Task 5 is an exception. Although the optimized marshalling model generates only 2 plans for it, but the optimal plan takes 45 minutes, which is more than 30 minutes for the one found by general marshalling model. That is because for Task 5, the optimized marshalling model finds the locally optimal solution, but not the globally optimal one.

The output of the model is the marshalling steps of the generated plans. According to the requirements in real work, as well as the defined symbols in Section 2 and Section 3 , the information of output can be organized and formatted into readable form. For example, it can be as:

Step 1: unhook carriage $C_{0}$;

Step 2: move carriage $C_{0}$ to Track $T_{2}$.

\section{Conclusions}

Based on the railway carriage information, track information, and marshalling task information, this paper constructs the state definitions which can fully express the realtime state information of railway stations, and abstract the marshalling problem into state space searching problem. A general marshalling model is built with the exhaustive method, which can be applied directly or be ground truth. Furthermore, greedy strategies are introduced to find the optimal solution more efficiently, which is verified by the experimental results, proving the feasibility and availability of the model.

Future work includes trying other searching and optimizing method such as evolutionary computation and multi-objective optimization for more complex constrains and states. In addition, researching on generating successive marshalling plans is under consideration. Implementing the models into standardized programs with standard inputs and outputs which can be easily used will be interesting, too.

\section{References}

[1] E. Dahlhaus, P. Horak, M. Miller, and J. F. Ryan, "The train marshalling problem," Discrete Applied Mathematics, 2000, vol. 103, no. 1-3, pp. 41-54.

[2] Dessouky M. Scheduling freight trains traveling on complex networks [J]. Transportation Research Part B, 2011, 45(7):0-1123.

[3] Krasemann, J. T. Greedy algorithm for railway traffic re-scheduling during disturbances: A Swedish case [J]. IET Intelligent Transport Systems, 2010, 4(4):375-0.

[4] He Z. Research on Improved Greedy Algorithm for Train Rescheduling[C]// Seventh International Conference on Computational Intelligence \& Security; 2011; Hainan, China: IEEE; p. 1197-1200.

[5] Pathania A , Venkataramani V , Shafique M, et al. Optimal Greedy Algorithm for Many-Core Scheduling[J]. IEEE Transactions on Computer-Aided Design of Integrated Circuits and Systems, 2016:1-1.

[6] Katsavounis S. A greedy algorithm for scheduling tasks on production lines[J]. Operational Research, 2001, 1(3):285-298.

[7] Ribas I, Companys R, Tort-Martorell X. An iterated greedy algorithm for the flowshop scheduling problem with blocking [J]. Omega, 2011, 39(3):293-301.

[8] Shi-Wei HE, Rui S , Fang L U, et al. Fuzzy Scheduling Problem with Multi-Processors Using Genetic Algorithm for Railway Management[J]. Journal of Beijing University of Aeronautics and Astronautics (Social Sciences Edition), 2000, 13(4):4-10. 
[9] Chuntian Zhang, Yuan Gao, Lixing Yang, et al. Joint optimization of train scheduling and maintenance planning in a railway network: A heuristic algorithm using Lagrangian relaxation[J]. Transportation Research Part B, 2020, 134(4): 64-92.

[10] Chuntian Zhang, Yuan Gao, Lixing Yang, et al. Integrated optimization of train scheduling and maintenance planning on high-speed railway corridors [J]. Omega, 2019, 87(6):86-104.

[11] Abels D., Jordi J., Ostrowski M., Schaub T., Toletti A., Wanko P. Train Scheduling with Hybrid ASP [C]. Logic Programming and Nonmonotonic Reasoning. LPNMR 2019. LNCS, (2019) vol 11481.

[12] Liu R, Li S, Yang L. Collaborative optimization for metro train scheduling and train connections combined with passenger flow control strategy[J]. Omega, 2020, 90: 101990. 